\title{
Byzacium, Byzacène
}

\section{J. Desanges}

\section{OpenEdition}

Journals

Édition électronique

URL : http://journals.openedition.org/encyclopedieberbere/1886

DOI : 10.4000/encyclopedieberbere.1886

ISSN : 2262-7197

\section{Éditeur}

Peeters Publishers

\section{Édition imprimée}

Date de publication : 1 septembre 1992

Pagination : 1674-1677

ISBN : 2-85744-581-4

ISSN : 1015-7344

\section{Référence électronique}

J. Desanges, «Byzacium, Byzacène », Encyclopédie berbère [En ligne], 11 | 1992, document B120, mis en ligne le 01 avril 2013, consulté le 25 septembre 2020. URL : http://journals.openedition.org/ encyclopedieberbere/1886 ; DOI : https://doi.org/10.4000/encyclopedieberbere.1886

Ce document a été généré automatiquement le 25 septembre 2020

(C) Tous droits réservés 


\section{Byzacium, Byzacène}

\section{J. Desanges}

1 Stéphane de Byzance (Ethn., s.v. Buzantes, éd. A. Meineke, p. 189) met en rapport un peuple des Buzantes*, situé non loin de Carthage, et une ville de Buzantion (cf. Buzakina, Ptol., IV, 3, 10, éd. C. Müller, p. 658 ; episcopus Bizaciensis, Gesta de 411,I, 198, "Sources chrétiennes », éd. S. Lancel, p. 854), qui lui doit son nom, avec une région appelée Busalas (sic) par Polybe au Livre XII, dans un contexte qui n'est pas parvenu jusqu'à nous. Bien que l'assimilation par Stéphane de Byzance de ces Buzantes aux Guzantes* d'Hérodote (IV, 194) soit suspecte, on peut admettre que leur ethnonyme est à l'origine des noms (Bussatis, Byzacium, Byzacène) qui ont servi à désigner un assez large territoire au sud de Carthage (cf. Buzakii*).

2 A consulter directement Polybe (III, 23, 2), on constate qu'il nommait cette région Bussatis, et non Busalas, forme certainement corrompue. Stéphane de Byzance luimême (Toc. cit.) l'appelle Buzakia, en remarquant que l'ethnique attendu est Buzakitēs [sous-entendu khōra (pays)], mais que l'on dit à présent Buzakeni. L'alternance* $k / t$ que l'on observe entre Bussatis et Buzakia devant une voyelle palatale ne saurait étonner.

3 Polybe, selon Stéphane de Byzance, considérait la Bussatis comme une région voisine des Syrtes, d'un périmètre de 2000 stades (soit quelque $355 \mathrm{~km}$, si l'on évalue le stade de Polybe à $178 \mathrm{~m}$ ) et d'une forme arrondie, ce qui, s'il s'agissait d'un cercle parfait, donnerait environ $115 \mathrm{~km}$ de diamètre. Par ailleurs (III, 23, 3), l'historien situe la Bussatis entre le Beau-Promontoire (presqu'île du Cap Bon) et la Petite Syrte. Le commentaire du premier traité entre Rome et Carthage, auquel il se livre, insiste sur la fertilité du pays, que les Puniques entendent soustraire à la convoitise des Romains. Cette fertilité sera par la suite affirmée pour le Byzacium (nom latin de cette région) par Varron (R., I, 44), Pline l'Ancien (V, 24 ; XVII, 41 ; XVIII, 94) et Silius Italicus (IX, 204-205).

4 Tite-Live (XXXIII, 48, 1) semble indiquer que lorsqu'on arrive sur la mer, en venant de Carthage, entre Acylla ( $\mathrm{H}^{\mathrm{r}}$ Botria) et Thapsus (Ras Dimasse), on a parcouru le Byzacium ; une nuit suffit pour en accomplir à cheval la traversée. Ptolémée, dont la documentation date au plus tard de Trajan, mentionne (IV, 3, 6, éd. C. Müller, p. 640) la région dite Buzakitis, au sud des Libyphéniciens, alors que Pline l'Ancien $(\mathrm{V}, 24)$ avait 
fait de ces Libyphéniciens les habitants du Byzacium. Mais l'orientation générale ouestest, à peine infléchie vers le sud, de la côte, de Neapolis (Nabeul) à Acholla $\left(\mathrm{H}^{\mathrm{r}}\right.$ Botria), dans la conception que s'en fait Ptolémée, donne à croire que la Buzakitis, pour lui, s'étend dans l'intérieur des terres, en arrière des villes côtières libyphéniciennes. Bien que lacunaire, l'article «Buzantes » de Stéphane de Byzance (loc. cit.) semble confirmer cette interprétation. En effet, Stéphane distingue deux parties dans la Buzakia, dont l'une à l'intérieur des terres appartient à Carthage, et deux sortes de Buzakeni, ou habitants de ces ensembles. Cette distinction doit dater de l'époque punique, alors que le statut des villes côtières était celui des cités libyphéniciennes, qui comportait des droits et des franchises, tandis que la plaine intérieure dépendait directement de l'administration carthaginoise.

5 Le Stadiasme de la Grande Mer (112-113, dans Geogr. Graec. Min., I, p. 469) considère Thapsus comme la limite entre le littoral de la Petite Syrte et la côte proprement punique. Inexacte géographiquement, cette distinction n'en est peut-être pas moins significative. Le Byzacium devait être à l'origine une région assez restreinte, dont la façade côtière s'étendait peut-être seulement de Thapsus à Neapolis (Nabeul). Le Stadiasme (113-117, p. 469-470) compte environ 610 stades entre ces deux villes, soit un peu moins de $115 \mathrm{~km}$. A l'intérieur des terres, le Byzacium était vraisemblablement borné par la Dorsale tunisienne. Sans doute englobait-il la steppe, pourtant mal irriguée, où s'élèvera Kairouan. Une autre hypothèse est que sa façade maritime partait du Ras Kaboudia pour aboutir à Pupput (Souk el-Abiod), localité qui, au Bas-Empire, fut située en Byzacène (Martianus Capella, VI, 670 ; I. L. Afr., 314), contrairement à Neapolis.

6 Une inscription de Madaure (I. L. Alg., I, 2025), postérieure à la mort de Gallien (268 de notre ère), mentionne le tractus Biz(acenus) englobant la regio Leptiminenf[sis], c'est-àdire la région de Lepti Minus (Lemta). Il s'agit d'une circonscription du patrimoine impérial qui préfigure la province de Byzacène (H.-G. Pflaum, «Une inscription de Madaure ", Rev. Soc. Arch. Constant, LXIX, 1955, p. 121-127), tout comme la regio Tripolitana, attestée dès le début du III ${ }^{e}$ siècle de notre ère, a précédé la province de Tripolitaine, créée sous la Tétrarchie (G. Di Vita-Evrard, «Regio tripolitana. A Reappraisal ", Town and Country in Roman Tripolitania. Papers in honour of Olwen Hackett, Oxford, 1985, p. 143-163). Il semble que ce soit en 303 après J.-C. (cf. Ead., dans L'Africa romana, II, Sassari, 1985, p. 149-177) que la province d'Africa fut partagée par la Tétrarchie en Zeugitane, Byzacène et Tripolitaine.

7 Outre Pupput, la nouvelle province de Byzacène (provincia Valeria Byzacena) comprenait, en partant de la côte au nord, les cités de Segermes ( $\mathrm{H}^{\mathrm{r}}$ Harat), Biia (Aïn-Batria) : cf. C.I.L., VIII, 11184, peut-être Abthugni ( $\mathrm{H}^{\mathrm{r}}$ Es-Souar) : cf. Cl. Lepelley, Les cités de l'Afrique romaine au Bas-Empire, II, Paris, 1981, p. 265-266, n. 5, Zama Regia (très probablement Jama) : C.I.L., VI, 1686, Thugga Terebentina ( $\mathrm{H}^{\mathrm{r}}$ Dougga); à l'ouest, Thala (Thala), mais non Ammaedara (Haïdra) : cf. A. Chastagnol et N. Duval, dans Mélanges W. Seston, Paris, 1974, p. 101-102; au sud-ouest, Cillium (Kasserine), Thelepte (Medinet el-Kdima) et Capsa (Gafsa) : cf. Procope, De aed., VI, 6, 18 ; Cod. Just., I, 26, 2, 1; sur la côte, au sud-est, Tacapes (Gabès) était en Tripolitaine, d'après un document, il est vrai, ecclésiastique ; en tout cas, Gightis (Bou Ghara) l'était assurément : cf. Itin. Marit., 518, 5, éd. O. Cuntz, p. 83.

8 La province de Byzacène, beaucoup plus étendue donc que le Byzacium punique, englobait plusieurs parties d'origine différente. La région côtière, jusqu'à Thaenae $\left(\mathrm{H}^{\mathrm{r}}\right.$ Thyna, au sud de Sfax) avait été territoire carthaginois jusqu'en 146 avant notre ère, puis avait appartenu à la Africa vetus jusqu'à la disparition de celle-ci au profit de la 
grande province d'Afrique, à l'époque d'Octave, ou au plus tard en 27 avant J.-C. La région intérieure, située à l'ouest de la Fossa regia (cf. Pline l'Ancien, V, 25 et le commentaire dans l'édition de la Coll. Univ. France, Paris, 1980, p. 241-242) était de tradition numide et le demeura (A. Chastagnol, «Les gouverneurs de Byzacène et de Tripolitaine», Ant. Afr., I, 1967, p. 120 ; S. Lancel, «Originalité de la province ecclésiastique de Byzacène aux IV et $\mathrm{V}^{\mathrm{e}}$ siècles ", Cah. de Tun., XII, n 45-46, 1964, p. 141-142 et 150). Au sud, dans la région des chotts, c'était le pays des Arzuges*, proche du limes et de la Tripolitaine. En bordure de la Petite Syrte, de Thaenae à Venefa ou Bennefa (Pline l'Ancien, XVI, 115 ; Ferrand, V. Fulg., 12 ; J. André dans Rev. de Philol, $3^{\mathrm{e}} \mathrm{s}$. XXXV, 1961, p. 52-53), au voisinage de Tacapes (Gabès), s'égrenaient les "emporia ", d'abord puniques, puis, pendant plus d'un siècle avant la campagne africaine de César, numides (R. Rebuffat, « Où étaient les Emporia ?», Semitica, XXXIX, 1990, p. 111-124).

Fort prospère, si l'on excepte certaines steppes de l'intérieur plus arides (cf. $\mathrm{Cl}$. Lepelley, Les cités de l'Afrique romaine au Bas-Empire, I, Paris, 1979, p. 29-49), avec une production d'huile considérable, qui ne supplantait pas entièrement de riches cultures céréalières ( $\mathrm{H}$. Slim, « Nouveaux témoignages sur la vie économique à Thysdrus (El Jem, Tunisie) », B.C. T.H., n.s. 19 B, 1983, p. 63-83), et des ateliers de céramique florissants (F. Zevi et A. Tchernia, "Amphores de Byzacène au Bas-Empire ", Ant. Afr., III, 1969, p. 173-214; A. Mahjoubi, J.W. Salomonson, A. Ennabli, La nécropole romaine de Raqqada, Tunis, fasc. 1 et 2, 1970 et 1973), la Byzacène connut la paix jusqu'en 439 ou 440 après J.$\mathrm{C}$, date à laquelle les Vandales l'annexèrent (Victor de Vita, I, 13). Ils l'occupèrent entièrement, jusqu'au Djerid, et même au delà, puisqu'ils firent de Turris Tamalleni (Telmine), un lieu de relégation pour les évêques récalcitrants (cf. Not. prov. et civ. Africae, Procos., 1, M.G.H.a.a., III/1, p. 63). Mais il ne semble pas que leur domination ait entraîné une décadence très sensible de la civilisation urbaine (cf. N. Duval, «Observations sur l'urbanisme tardif de Sufetula (Tunisie) », Cah. de Tun., XII, n 45-46, 1964, p. 87-103). Cependant nombre de campagnes, épargnées depuis des siècles par les insurrections indigènes, furent ravagées pendant une vingtaine d'années (529-548) par les « Maures », tribus insoumises à l'autorité vandale d'abord, puis, à partir de 534, à l'autorité byzantine. Hadrumète même fut pillée dans l'été de 544 (Procope, B. V., II, 23, 11-16). Par la suite, les insurrections berbères, qui sévirent à plusieurs reprises dans l'Afrique byzantine, n'épargnèrent certainement pas la Byzacène; mais les sources nous renseignent très mal à ce sujet (cf. Y. Modéran, De bellis Libycis. Berbères et Byzantins en Afrique au VI e siècle, quatrième partie, à paraître dans la B.E.F.A.R.).

On ne sait si les Byzantins ont réussi à contrôler le sud-ouest de la Byzacène. Ch. Diehl (L'Afrique byzantine, Paris, 1896, p. 232-234) supposait que la frontière de la province suivait au sud la voie Tacapes (restée en Tripolitaine, cf. Procope, De aed., VI, 4, 14) Capsa (Gafsa), puis «remontait brusquement » vers le nord, pour atteindre Thelepte (Medinet el-Kdima), et ensuite Cillium (Kasserine), d'où elle rejoignait, en Numidie, Theveste (Tebessa). Mais on a admis plus récemment que les Byzantins ont pu, au moins pendant un certain temps, occuper Tusuros (Tozeur) et l'ensemble d'oasis que les Arabes appelleront "Qastiliya », d'une part, et, d'autre part, Ad Majores $\left(\mathrm{H}^{\mathrm{r}}\right.$ Besseriani, près de Négrine) en Numidie (Chr. Courtois, Les Vandales et l'Afrique, Paris, 1955, p. 326, n. 8 ; P. Trousset, «Les fines antiquae et la reconquête byzantine en Afrique », B.C.T.H., n.s. 19 B, 1983, p. 375). En tout cas, il semble qu'en 546, pour accroître les capacités de défense de la province, le commandement des troupes qui y étaient stationnées fut divisé entre deux duces (Corippus, Joh., VI, 49), dont l'un devait veiller plus 
particulièrement sur le littoral, et l'autre sur les régions intérieures (Ch. Diehl, op. cit., p. 472). Le premier siégeait peut-être à Hadrumète (Corippus, Joh., PV, 8), tandis que le second semble avoir eu un double quartier général, à Capsa et à Thelepte (Cod. Just., I, 27, 2, 1 a ; D. Pringle, The Defence of Byzantine Africa from Justinian to the Arab Conquest, I, Oxford, 1981, p. 63). Cette dichotomie de la Byzacène entre une zone maritime et une zone intérieure, qui nous ramène curieusement à l'époque punique évoquée par Stéphane de Byzance à propos du pays des Byzantes*, structure le développement que Procope (De aed., VI, 6, 17, notamment) consacre aux fortifications de la province.

En 647, les Arabes commencèrent à piller l'intérieur de la Byzacène. En s'opposant à leur incursion, le patrice Grégoire, qui gouvernait l'Afrique au nom de Byzance, fut défait et tué dans la région de Sufetula (Sbeida). La ville elle-même fut prise d'assaut et pillée. Capsa et le Djerid furent dévastés. Les Arabes se retirèrent bientôt, mais les populations berbères du sud de la Byzacène semblent dès lors avoir pris leurs distances à l'égard du pouvoir byzantin. La province fut encore razziée en 665 . Puis en 669 , Okba ibn Nafi, après s'en être pris à nouveau à Capsa, parvint dans la steppe où il allait construire pendant cinq ans (670-675) Kairouan. La Byzacène était perdue pour les Byzantins une trentaine d'annés avant que les Arabes ne prissent définitivement Carthage (698 de notre ère). La présence d'une forte garnison arabe à Kairouan, aux frontières de la Proconsulaire, a scellé le destin de l'Afrique byzantine (Ch. Diehl, op. cit., p. 574). Quant à la brève reconquête de Kairouan (683-688 environ) par les Berbères de Koçéila, ce fut une entreprise indépendante de l'administration byzantine, et d'ailleurs sans lendemain.

\section{BIBLIOGRAPHIE}

En l'absence d'une monographie sur le Byzacium et sur la Byzacène, on consultera surtout :

COURTOIS Ch., Les Vandales et l'Afrique, Paris, 1955.

DESANGES J., « Étendue et importance du Byzacium avant la création, sous Dioclétien, de la province de Byzacène ", Cahiers de Tunisie, XI, n 44, 1963, p. 7-22.

DIEHL Ch., L'Afrique byzantine, Paris, 1896.

LANCEL S., " Originalité de la province ecclésiastique de Byzacène aux IV et v viècles », Cahiers de Tunisie, XII, nº 45-46, 1964, p. 139-153, une carte h.t. (la Byzacène ecclésiastique).

LEPELLEY Cl., Les cités de l'Afrique romaine au Bas-Empire, I et II, Paris, 1979 et 1981, cf. particulièrement II, p. 259-331 (« Notices d'histoire municipale : province de Byzacène »).

MODÉRAN Y., De bellis Libycis. Berbères et Byzantins en Afrique au vie siècle (à paraître dans la Bibl. des Écoles françaises d'Athènes et de Rome).

PRINGLE D., The Defence of Byzantine Africa from Justinian to the Arab Conquest (= B.A.R., intern. séries 99, 1 et 2), Oxford, 1981. 
INDEX

Mots-clés : Antiquité, Tunisie 